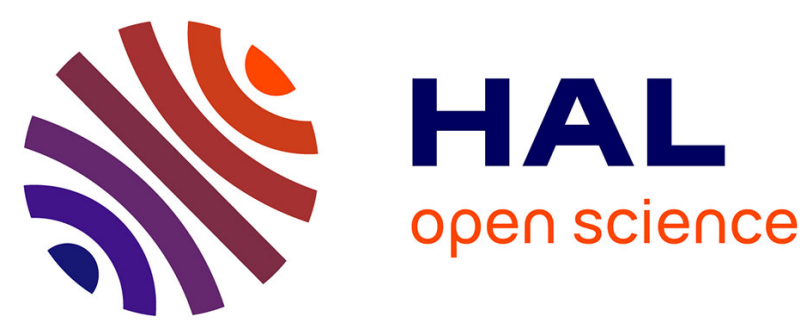

\title{
STATE ESTIMATION FOR WASTEWATER TREATMENT PROCESSES
}

Olivier Bernard, Benoit Chachuat, Jean-Philippe Steyer

\section{To cite this version:}

Olivier Bernard, Benoit Chachuat, Jean-Philippe Steyer. STATE ESTIMATION FOR WASTEWATER TREATMENT PROCESSES. Philippe Quevauviller. Wastewater Quality Monitoring and Wastewater Quality Monitoring and Treatment, 6 (6), John Wiley \& Sons Ltd, 2006, Wastewater Monitoring; Water Quality Measurements, 10: 0-471-49929-3. inria-00122496

\section{HAL Id: inria-00122496 https://hal.inria.fr/inria-00122496}

Submitted on 3 Jan 2007

HAL is a multi-disciplinary open access archive for the deposit and dissemination of scientific research documents, whether they are published or not. The documents may come from teaching and research institutions in France or abroad, or from public or private research centers.
L'archive ouverte pluridisciplinaire HAL, est destinée au dépôt et à la diffusion de documents scientifiques de niveau recherche, publiés ou non, émanant des établissements d'enseignement et de recherche français ou étrangers, des laboratoires publics ou privés. 


\title{
STATE ESTIMATION FOR WASTEWATER
}

\section{TREATMENT PROCESSES}

\author{
O. Bernard ${ }^{1}$, B. Chachuat ${ }^{2}$, and J.-Ph. Steyer ${ }^{3}$ \\ ${ }^{1}$ INRIA Comore, 2004 Route des Lucioles, B.P. 93, 06902 Sophia-Antipolis Cedex, France (e-mail: \\ Olivier.Bernard@inria.sophia.fr) \\ ${ }^{2}$ EPFL, Automatic Control Laboratory, Station 9, 1015 Lausanne, Switzerland (e-mail: \\ benoit.chachuat@epfl.ch) \\ 3 INRA-LBE, Avenue des Étangs, 11100 Narbonne, France (e-mail: steyer@ensam.inra.fr)
}

\begin{abstract}
In this chapter, we provide the key ideas on how to build software sensors (also called observers) for wastewater treatment plants (WWTPs). We give an overview of the existing linear and nonlinear observers and discuss criteria that help to identify which observer is best suited with respect to the amount of information being available for the WWTP. Depending on the model reliability, the available measurements and the level of uncertainties associated to the influent concentrations, different class of observers can be considered. We distinguish between those that rely on a full model description (e.g., the
\end{abstract}


extended Kalman filter), and those based on a mass-balance model wherein the biological kinetics are assimilated to unknown inputs (e.g., the asymptotic observer). Moreover, if bounds are known for the uncertainties, then interval observers can be designed. We discuss the principles of each class of observers and illustrate them through a number of examples.

\subsection{INTRODUCTION}

A major bottleneck in the application of advanced monitoring and optimization strategies for WWTPs lies in the difficulty of measuring chemical and biological variables. Even though considerable progress in on-line sensors technology has been made over the recent years, it is still often difficult to measure many of the key state variables such as biomass concentration, specific bacterial activity or intermediate products concentration. Hence, the idea of using observers, also called software sensors, that combine a number of readily available on-line measurements (gaseous flow rates, $\mathrm{pH}$, dissolved gases, etc.) with a process model for estimating the values of (unmeasured) state variables.

Quite of few methods have been proposed to design such observers. In this chapter, we shall only focus on those approaches that are relevant to the field of WWTPs. It is worthwhile noting that the principles underlying the design of observers can be quite different. Therefore, the choice of an observer inherently depends on the specificities of the problem at hand. In practice, this choice is strongly guided by the reliability of the process model as well as the amount and accuracy of the data. If a reliable process model is available and if this model has been thoroughly identified and validated, either an (extended) Kalman filter or a high gain observer can be developed. When the process model is not accurate enough, an asymptotic observer relying on mass-balance principles, but not on the uncertain kinetics, shall be used instead. Finally, If bounds are known for the uncertain inputs and/or parameters, an interval observer can be used for predicting intervals in which the unmeasured variable are guaranteed to belong (instead of pointwise estimates).

The type of observer to be constructed should not only be based on the model quality, but it must also account for the objectives to be achieved. Indeed, an observer can have other purposes than simply monitoring a WWTP. It can be developed with the objective of 
applying a control action that needs an estimate of some internal state; it can also be used for diagnosing whether a failure occurred during process operation or not.

The remainder of this chapter is organized as follows. A number of useful definitions and results are given in section 1.2. Observers that require a full-model description of the process are presented in section 1.3, with emphasis placed on linear systems. The design of observers relying on the mass-balance principles is discussed in section 1.4. Interval observers that exploit knowledge of bounds on the model uncertainty are presented in section 1.5. Finally, section 1.6 concludes the chapter.

\subsection{PRELIMINARIES}

This section gives an overview of the main theoretical concepts in system observability. These concepts are useful in the analysis conducted later on. The interested reader is referred to $[8,17]$ for additional information.

It is first fundamental to study the observability property of a system prior to designing an observer. Intuitively, observability consists of determining whether the measured signals contain sufficiently rich information to estimate the unmeasured state variables; a system is then said to be observable if it satisfies this property from a theoretical point of view. The problem to address next is to derive an observer for the problem at hand, i.e. an auxiliary dynamic system that provides the state estimates. At this point, it should be noted that the problems of observability and observer design are very different in nature. In particular, the observability property does not give any clue on how to build an observer.

The theory of observation has been extensively developed in the linear case. Several methods also exist in the nonlinear case, but are tailored to specific classes of models.

\subsubsection{Notion of Observability}

We consider the following general model driving the process dynamics:

$$
\left\{\begin{array}{l}
\frac{d x(t)}{d t}=f(x(t), u(t)) ; \quad x(0)=x_{0} \\
y(t)=h(x(t))
\end{array}\right.
$$


where $u \in \mathbb{R}^{m}$ is the input vector; $y \in \mathbb{R}^{p}$, the output vector; $x \in \mathbb{R}^{n}$, the state vector made-up of the concentrations of the various species inside the liquid phase; and $x_{0}$, the vector of initial conditions. The applications $f$ and $h$ provide the dynamics of the state variables and the links between the state variables and the measurements, respectively.

The objective is to estimate $x(t)$ from the measurements $y(t)$. Observability is a structural property of a system that states whether this is possible or not.

Property 1 The system $(\mathcal{S})$ is said to be observable, if $x(t)$ can be uniquely determined from $y(t), \frac{d y(t)}{d t}, \frac{d^{2} y(t)}{d t^{2}}, \ldots, \frac{d^{n} y y(t)}{d t^{n} y}$, and $u(t), \frac{d u(t)}{d t}, \frac{d^{2} u(t)}{d t^{2}}, \ldots, \frac{d^{n} u(t)}{d t^{n} u}$, for some (possibly infinite) $n_{y} \geq 0$ and $n_{u} \geq 0$.

The reader is referred to $[8,17]$ for more details.

\subsubsection{General Definition of an Observer}

Once a system has been shown to be observable, the next step is to design an observer that estimates the state variable $x$ based on a model and a set of input/output measurements. The principle of an observer is presented in Fig. 1.1. Roughly speaking, an observer is an auxiliary dynamic system coupled to the original system via the measured inputs and outputs. This is formalized in the following definition.

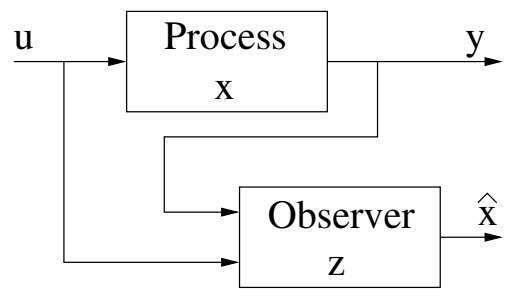

Figure 1.1. Observer principle

Definition 1 An observer is an auxiliary system $(\mathcal{O})$ coupled to the original system $(\mathcal{S})$ as:

$$
\left\{\begin{array}{l}
\frac{d z(t)}{d t}=\hat{f}(z(t), u(t), y(t)) ; z(0)=z_{0} \\
\hat{x}(t)=\hat{h}(z(t), u(t), y(t))
\end{array}\right.
$$


where $z \in \mathbb{R}^{q}$ denotes the state of the observer; $\hat{f}$ is the observer dynamics; and $\hat{h}$ relates $z$ to the estimate $\hat{x}$ of the real system. An observer has the property that the observation error converges to zero asymptotically:

$$
\lim _{t \rightarrow \infty}\|\hat{x}(t)-x(t)\|=0 .
$$

A desirable property for an observer is the ability to tune the convergence rate in order for the estimates to converge more rapidly than the original dynamics of the system. Another desirable property is that the estimate $\hat{x}(t)$ should remain equal to $x(t)$ under proper initialization, i.e. when it is initialized with the true value $x(0)$. This easily justifies that following structure is often used to design observers in practice:

$$
\left\{\begin{aligned}
\frac{d \hat{x}(t)}{d t} & =f(\hat{x}(t), u(t))+k[z(t), h(\hat{x}(t))-y(t)] \\
\frac{d z(t)}{d t} & =\hat{f}(z(t), u(t), y(t)) \quad \text { with } \quad k(z(t), 0)=0 .
\end{aligned}\right.
$$

This observer consists of a replica of the original dynamics corrected by a term that depends on the discrepancy between both the measured and predicted outputs. Note also that the correction amplitude is tuned via the function $k$ that is often referred to as the observer gain (internal tuning of the observer).

\subsection{OBSERVERS FOR LINEAR SYSTEMS}

For time-invariant, linear systems, the general system $(\mathcal{S})$ simplifies to

$$
\left\{\begin{aligned}
\frac{d x(t)}{d t} & =A x(t)+B u(t) \\
y(t) & =C x(t)
\end{aligned}\right.
$$


with $A \in \mathbb{R}^{n \times n}(n \geq 2)$ and $C \in \mathbb{R}^{p \times n}$. A well known observability criterion for $\left(\mathcal{S}_{\ell}\right)$ is given by the rank condition:

$$
\operatorname{rank}\left(\begin{array}{c}
C \\
C A \\
\vdots \\
C A^{n-1}
\end{array}\right)=n \text {. }
$$

\subsubsection{Luenberger Observer}

Theorem 1 If the pair $(A, C)$ is observable, a Luenberger observer for $\left(\mathcal{S}_{\ell}\right)$ is obtained as [16]

$$
\frac{d \hat{x}(t)}{d t}=A \hat{x}(t)+B u(t)+K(C \hat{x}(t)-y(t))
$$

where $K$ is a $n \times n$ gain matrix that can be used for tuning the convergence rate of the observer, and can be chosen in order for the observation error to converge to zero arbitrarily fast.

Proof. The dynamics of the observation error $e(t)=\hat{x}(t)-x(t)$ is given by

$$
\frac{d e}{d t}=(A+K C) e
$$

and is independent of the input $u(t)$. The result follows from the pole placement theorem which guarantees that the error dynamics can be chosen arbitrarily.

In principle, the gain matrix $K$ can be chosen in such a way that the observation error converges to zero as quickly as desired. However, the larger the gain of the observer, the more sensitive it becomes to external perturbations (measurement noise for example). A good compromise must thus be sought that ensures both stability and accuracy at the same time. The Kalman filter, discussed in subsection 1.3.3 below, proposes a way of achieving such a compromise. 


\subsubsection{The Linear Case up to an Output Injection}

A particular situation wherein a linear observer can be designed for a nonlinear system arises in the simple case where the nonlinearities depends on the output $y$ only:

$$
\left\{\begin{aligned}
\frac{d x(t)}{d t} & =A x(t)+\phi(t, y(t))+B u(t) \\
y(t) & =C x(t)
\end{aligned}\right.
$$

with $\phi$ being a (known) nonlinear function in $\mathbb{R}^{n}$. The following "Luenberger-like" observer has linear dynamics with respect to the observation error:

$$
\frac{d \hat{x}(t)}{d t}=A \hat{x}(t)+\phi(t, y(t))+B u(t)+K(C \hat{x}(t)-y(t)) .
$$

In particular, the error dynamics can be chosen arbitrarily, provided that the pair $(A, C)$ is observable. Here again, however, an adequate choice for the gain vector $K$ is one that guarantees a fast enough convergence of the observer, while keeping it stable.

\subsubsection{Kalman Filter}

The Kalman filter is notorious in the field of linear systems (see e.g. [15]). Loosely speaking, a Kalman filter can be seen as a Luenberger observer with a time varying gain. More specifically, the gain is chosen in such a way that the variance of the observation error is minimized (or, equivalently, the integral between $t_{0}$ and $t$ of the squared errors is minimized); for this reason the Kalman filter is often referred to as the optimal estimator.

Consider an observable continuous-time system in the following stochastic representation:

$$
\frac{d x(t)}{d t}=A x(t)+B u(t)+G w(t) ; x\left(t_{0}\right)=x_{0}
$$

where $w(t) \sim(0, Q(t))$ is a white noise process with zero mean and covariance $Q(t)$. Suppose that initial state $x_{0}$ is unknown, but there is available a priori knowledge that 
$x_{0} \sim\left(\bar{x}_{0}, P_{0}\right)$. Suppose also that measurements are given at discrete times $t_{k}$ according to

$$
y_{k}=C x\left(t_{k}\right)+v_{k}
$$

where $v_{k} \sim\left(0, R_{k}\right)$ is uncorrelated with $w(t)$ and $x_{0}$.

Besides initialization, a continuous/discrete Kalman filter for system $(1.1,1.2)$ consists of two steps: a propagation step (between two successive measurements), followed by a correction step (at measurement times):

$$
\begin{aligned}
& \text { Initialization }\left(t=t_{0}^{+}\right) \text {: } \\
& P\left(t_{0}\right)=P_{0}, \hat{x}\left(t_{0}\right)={ }^{-} x_{0} \\
& \text { Propagation }\left(t_{k-1}^{+} \leq t \leq t_{k}^{-}, k \geq 1\right): \\
& \frac{d P(t)}{d t}=A P(t)+P(t) A^{T}+G Q(t) G \\
& \frac{d x(t)}{d t}=A \hat{x}(t)+B u(t) \\
& \text { Correction }\left(t=t_{k}^{+}, k \geq 1\right): \\
& K_{k}=P\left(t_{k}^{-}\right) C^{T}\left[C P\left(t_{k}^{-}\right) C^{T}+R_{k}\right]^{-1} \\
& P\left(t_{k}^{+}\right)=\left(I-K_{k}(t)\right) P\left(t_{k}^{-}\right) \\
& x\left(t_{k}^{+}\right)=x\left(t_{k}^{-}\right)+K_{k}\left(z_{k}-C x\left(t_{k}^{-}\right)\right)
\end{aligned}
$$

At this point, we shall emphasize on several points. Note first that the foregoing Kalman filter can be applied to time-varying linear system, i.e. with matrices $A, B, C$ and $G$ depending on time. One should however keep in mind that observability must be proven for such systems prior to constructing the observer. Note also that Kalman filters can be extended by adding a term $-\theta P(t), \theta>0$, in the propagation equation of $P$. This exponential forgetting factor allows us to consider the case where $Q=0$. Finally, estimating the positive definite matrices $R, Q$ and $P_{0}$ often proves to be tricky in practice, especially when the noise properties are not known precisely. 


\subsubsection{The Extended Kalman Filter}

Consider a continuous-time nonlinear system of the form

$$
\frac{d x(t)}{d t}=f(x(t))+G(t) w(t) \quad ; \quad x\left(t_{0}\right)=x_{0}
$$

with measurements at discrete time $t_{k}$ given by

$$
y_{k}=h\left(x\left(t_{k}\right)\right)+v_{k} .
$$

The idea behind Extended Kalman Filter $(\mathrm{EKF})$ is to linearize the nonlinear system $(1.3,1.4)$ around its current state estimate $\hat{x}(t)$ [15]. By doing so, the problem becomes equivalent to building a Kalman filter for a non-stationary linear system $(1.1,1.2)$ with $A$ and $C$ taken as

$$
A(t)=\left.\frac{\partial f}{\partial x}\right|_{\hat{x}(t)}, \quad C\left(t_{k}\right)=\left.\frac{\partial h}{\partial x}\right|_{\hat{x}\left(t_{k}\right)} .
$$

The EKF is used routinely and successfully in many practical applications, including WWTPs, even though few theoretical guarantees can be given as regards its convergence $[2,15]$. Note also that multirate versions of the EKF have been developed to handle those (rather frequent) situations where measurements are available at different samplings rates [10]. An application of EKF in alternating activated sludge WWTPs is detailed hereafter. Other applications to the activated sludge process can be found, e.g. in [18, 23].

\subsubsection{Application to an Alternating-Activated-Sludge Plant}

We consider an alternating-activated-sludge (AAS) WWTP similar to the one shown in Fig. 1.2. AAS plants degrade both organic and nitrogenous compounds by alternating aerobic and anoxic phases in the bioreactor. Besides dissolved oxygen (DO) concentration that is routinely measured in activated sludge WWTPs, both nitrate (NO) and ammonia (NH) concentrations can also be measured on-line (at a lower frequency than DO, though). The objective here is to estimate the concentration of COD in the bioreactor based on these measurements. 


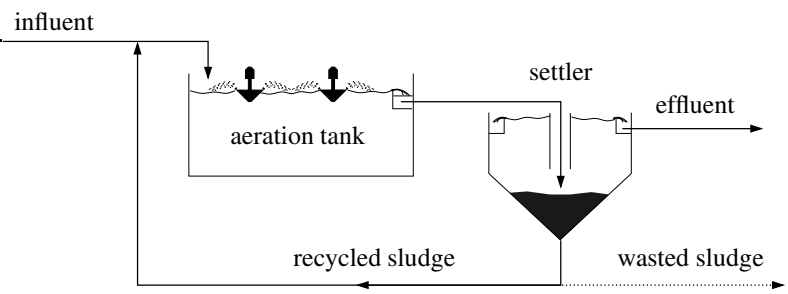

Figure 1.2. Typical small-size alternating activated sludge treatment plant.

A multirate EKF is developed based on the reduced nonlinear model given in [6]. This 5-state model describes the dynamics of COD, NO, NH, organic nitrogen (ND) and DO, and was shown to be observable under both aerobic and anoxic conditions (with the aforementioned measurements).

Numerical simulations have been performed by using a set of synthetic data produced from the full ASM1 model [12] corrupted with white noise. The DO, NO and NH measurements are assumed to be available every $10 \mathrm{sec} ., 10 \mathrm{~min}$. and $10 \mathrm{~min}$, , respectively. The results are shown in Fig. 1.3.; for the sake of comparison, the EKF estimates are compared to the open-loop estimates (i.e., without correction).
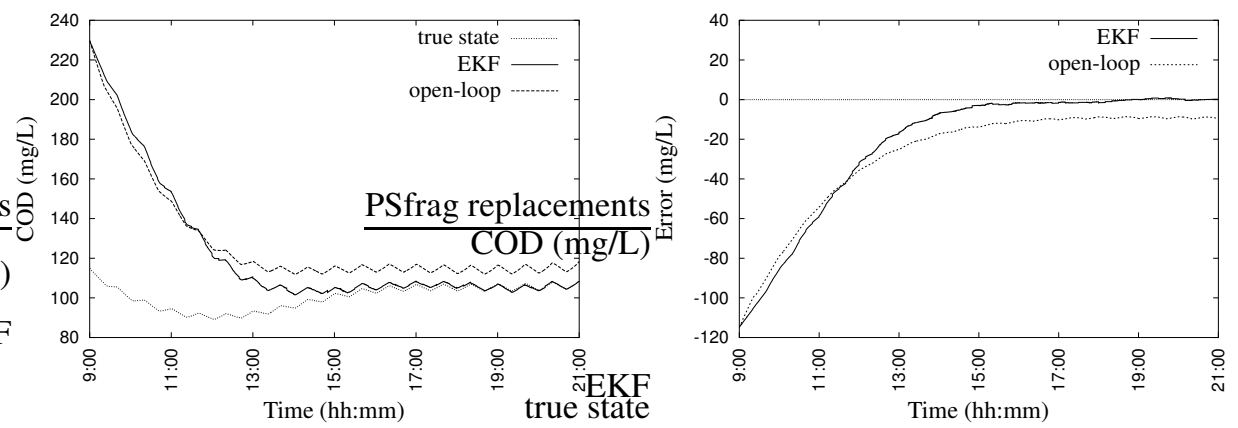

Figure 1.3. Estimated COD concentration (left plot) and observation error (right plot).

These results show satisfactory performance of the EKF for COD estimation. However, it should be noted that the COD estimates are very sensitive to model-parameter mismatch, which is hardly compatible with the fact that some parameters are time-varying and/or badly known in real applications. This motivates the development of mass-balance-based observer that are independent of the uncertain kinetic terms. 


\subsection{OBSERVERS FOR MASS-BALANCE-BASED SYSTEMS}

The underlying structure of many WWTP models consists of two parts [2]: (i) a linear part based on mass-balance considerations; and (ii) a number of nonlinear term that describes the biological reaction rates (kinetics). These latter kinetic terms are often poorly known in practice, and there is little hope to construct a reliable observer by accounting for such uncertain terms. In contrast to the previous section wherein a full-model structure was used in the observer design, we shall show, in this section, how to take advantage of the foregoing twofold structure to design observers that are independent of the uncertain reaction rates, via a linear change of variables. As we shall see, the main condition to design a so-called asymptotic observer is that enough variables are measured. Note also asymptotic observers fall into the broad class of observers with unknown inputs [7, 13, 14], whose principle relies on cancellation of the unknown part via a change of variables.

\subsubsection{Preliminaries}

Throughout this section, we consider mass-balance models for WWTPs of the following form [2]:

$$
\frac{d x}{d t}=K r(x)-D(t) x+D(t) x^{\mathrm{in}}(t)-Q(x)
$$

where $x, x^{\text {in }} \in \mathbb{R}^{n}$ represent the concentrations in the reactor and the influent, respectively; $D \in \mathbb{R}^{n \times n}$, the dilution rate matrix; $Q(x) \in \mathbb{R}^{n}$, the gaseous exchange between the reaction medium and the environment; $r(x) \in \mathbb{R}^{p}$, the reaction rates; and $K \in \mathbb{R}^{n \times p}$, a constant pseudo-stoichiometric coefficient matrix. In this representation, $K r(x)$ stands for the biological and biochemical conversions in the reactor (per unit of time) according to the underlying macroscopic reaction network.

Suppose that the set of available measurements $y$ corresponds to is partitionned into $y_{1}$ and $y_{2}$ such that:

- $y_{1}$ is a set of $q \leq n$ measured state variables; without loss of generality, we assume that $y_{1}$ corresponds to the first $q$ components of $x, y_{1}=\left[x_{1}, \ldots, x_{q}\right]^{T}$.

- $y_{2}$ consists of the measured gaseous flow rates, $y_{2}=\left[q_{1}(x), \ldots, q_{n}(x)\right]^{T}$. 
The measurements $y_{1}$ induce a partition of the state variables $x=\left[x_{1}, x_{2}\right]$ with $x_{1}=y_{1}$. Accordingly, Eq. (1.5) can be rephrased as

$$
\begin{aligned}
& \frac{d x_{1}}{d t}=K_{1} r(x)-D x_{1}+D x_{1}^{\mathrm{in}}-Q_{1}(x) \\
& \frac{d x_{2}}{d t}=K_{2} r(x)-D x_{2}+D x_{2}^{\mathrm{in}}-Q_{2}(x)
\end{aligned}
$$

where matrices $K_{1}$ and $K_{2}$, vectors $x_{1}^{\text {in }}, x_{2}^{\text {in }}, q_{1}$ and $q_{2}$ are such that

$$
K=\left(\begin{array}{c}
K_{1} \\
K_{2}
\end{array}\right), x^{\text {in }}=\left(\begin{array}{c}
x_{1}^{\text {in }} \\
x_{2}^{\text {in }}
\end{array}\right), q=\left(\begin{array}{l}
q_{1} \\
q_{2}
\end{array}\right)
$$

\subsubsection{Asymptotic Observers}

Constructing an asymptotic observers for system (1.5) requires the following two technical assumptions to hold.

Assumption 1 (i) There are more measured quantities than reactions, i.e. $q \geq p$; (ii) Matrix $K_{1}$ has full rank.

These assumptions guarantee that a non-zero $r$ cannot cancel the term $K_{1} r$ and the $q \times p$ matrix $K_{1}$ has a left inverse. Accordingly, there exists a $p \times q$ matrix $G$ such that:

$$
G K_{1}=I_{p}
$$

Let us denote $A=-K_{2} G$ and $M=\left(\begin{array}{ll}A & I_{n-p}\end{array}\right)$. An observer for subsystem (1.6) can be obtained as indicated in the following lemma.

Lemma 1 (see [2]) If $D$ is positive definite, the solution $\hat{x}_{2}$ of the auxiliary system

$$
\begin{aligned}
\frac{d \hat{\zeta}_{2}}{d t} & =-D\left(\hat{\zeta}_{2}-M x^{i n}\right)-M y_{2} \\
\hat{x}_{2} & =\hat{\zeta}_{2}-A y_{1}
\end{aligned}
$$

converges to the solution $x_{2}$ of subsystem (1.6), asymptotically. 


\subsubsection{Application to an Anaerobic Digester}

We consider a very simple model of the anaerobic digestion process that accounts for a single "global" degradation step of the soluble COD $\left(S_{t}\right)$ by the biomass $X_{t}[1]$ :

$$
k_{t} S_{t} \stackrel{\mu_{t}(.) X_{t}}{\longrightarrow} X_{t}+k_{m} \mathrm{CH}_{4}
$$

where $k_{t}$ and $k_{m}$ are the yield coefficients associated to COD degradation and methane production, respectively; $\mu_{t}(\cdot)$ stands for bacterial growth rate. The corresponding massbalance model reads:

$$
\begin{aligned}
\frac{d S_{t}}{d t} & =-k_{t} \mu_{t}(\cdot) X_{t}-D\left(S_{t}-S^{\text {in }}\right) \\
\frac{d X_{t}}{d t} & =\mu_{t}(\cdot) X_{t}-\alpha D X_{t} \\
q_{\mathrm{CH}_{4}} & =k_{m} \mu_{t}(\cdot) X_{t}
\end{aligned}
$$

where $q_{\mathrm{CH}_{4}}$ stands for the methane outflow rate; $D$, the dilution rate; and $\alpha$, the fraction of bacteria not attached onto a support (i.e., being affected by the dilution rate $D$ in the reactor).

The objective is to design an asymptotic observer for the biomass $X_{t}$, based on COD measurements, without knowing the reaction rates $\mu_{t}(\cdot)$. In this case, we have $x_{1}=y_{1}=$ $S_{t}, x_{2}=X_{t}$ and $Q(x)=0$. Moreover, $K_{1}=-k_{t}$ and $K_{2}=1$, hence a possible choice for matrices $A$ and $G$ is: $G=-1 / k_{t}, A=1 / k_{t}$. Finally, lemma 1 provides the following auxiliary differential system:

$$
\begin{aligned}
\frac{d \hat{\zeta}_{2}}{d t} & =-\alpha D\left(\hat{\zeta}_{2}-\frac{S^{\text {in }}}{\alpha k_{t}}\right)-(1-\alpha) D \frac{y_{1}}{k_{t}} \\
\hat{X}_{t} & =\hat{\zeta}_{2}-\frac{y_{1}}{k_{t}} .
\end{aligned}
$$

Eqs. (1.9)-(1.10) is an observer for the biomass concentration in the digester. However, this observer may provide poor estimates in practice, because of the large errors made on 
$S^{\text {in }}$ and $k_{t}$. A more appropriate solution is then to use an interval observer for coping with uncertainty, as discussed in the following section.

\subsection{INTERVAL OBSERVERS}

Usual observers rely on the implicit assumption that the process model is a good approximation of the real plant. Nevertheless, we have seen that WWTP models are often corrupted. In such situations where large modeling and measurement errors prevail, one can no longer construct an exact observer (i.e., with the guarantee that the observation error converges to zero asymptotically and that the convergence rate can be tuned). Instead, the observation principle must be weakened. In this section, we explain how to derive rigorous bounds enclosing the estimated states by accounting for the uncertainty in the process model $[9,19]$.

\subsubsection{Principle}

Interval observers require knowledge of time-varying bounds enclosing the uncertainty, $v^{-}(t) \leq v(t) \leq v^{+}(t)$. These bounds are used to calculate time-varying bounds enclosing the state variable to be estimated.

Consider the following general system:

$$
\left\{\begin{aligned}
\frac{d x(t)}{d t} & =f(x(t), u(t), w(t)) \quad ; \quad x\left(t_{0}\right)=x_{0} \\
y(t) & =h(x(t), v(t))
\end{aligned}\right.
$$

where known lower and upper bounds are available for the uncertain quantities $w \in \mathbb{R}^{r}$ and $v \in \mathbb{R}^{s}$,

$$
\begin{gathered}
w^{-}(t) \leq w(t) \leq w^{+}(t) \quad \forall t \geq t_{0} \\
v^{-}(t) \leq v(t) \leq v^{+}(t) \quad \forall t \geq t_{0} .
\end{gathered}
$$

Based on the fixed model structure $\left(\mathcal{S}_{\mathcal{I}}\right)$ and on the set of measured data, an auxiliary dynamic system $\left(\mathcal{O}_{\mathcal{I}}\right)$ can be designed such that it provides a lower bound and an upper 
bound for the state variables:

$$
\left\{\begin{aligned}
\frac{d z^{-}}{d t} & =f^{-}\left(z^{-}, z^{+}, u, y, w^{-}, w^{+}, v^{-}, v^{+}\right) ; z^{-}\left(t_{0}\right)=g^{-}\left(x_{0}^{-}, x_{0}^{+}\right) \\
\frac{d z^{+}}{d t} & =f^{+}\left(z^{-}, z^{+}, u, y, w^{-}, w^{+}, v^{-}, v^{+}\right) ; z^{+}\left(t_{0}\right)=g^{+}\left(x_{0}^{-}, x_{0}^{+}\right) \\
x^{-} & =h^{-}\left(z^{-}, z^{+}, u, y, w^{-}, w^{+}, v^{-}, v^{+}\right) \\
x^{+} & =h^{+}\left(z^{-}, z^{+}, u, y, w^{-}, w^{+}, v^{-}, v^{+}\right) .
\end{aligned}\right.
$$

Definition 2 (interval estimator) System $\left(\mathcal{O}_{\mathcal{I}}\right)$ is said to be an interval estimator of system $\left(\mathcal{S}_{\mathcal{I}}\right)$ if for any pair of initial conditions $x^{-}\left(t_{0}\right) \leq x\left(t_{0}\right) \leq x^{+}\left(t_{0}\right)$, there exists bounds $z^{-}\left(t_{0}\right), z^{+}\left(t_{0}\right)$ such that the solutions of the coupled system $\left(\mathcal{S}_{\mathcal{I}}, \mathcal{O}_{\mathcal{I}}\right)$ verifies:

$$
x^{-}(t) \leq x(t) \leq x^{+}(t) \quad \forall t \geq t_{0} .
$$

Interval estimators result from the coupling of two estimators which provide both an underestimate $x^{-}(t)$ and an overestimate $x^{+}(t)$ of $x(t)$. Of course, such bounds can be very large, thus making interval observers practically useless in some cases. However, for particular classes of systems (e.g., linear systems up to an output injection), theoretical guarantees can be given that the time-varying intervals $\left[x^{-}(t), x^{+}(t)\right]$ converge to a "limit" interval of finite magnitude [9]. Moreover, the convergence rate towards this limit interval can be tuned if certain properties hold [20]. Note that these ideas find their origin in the theory of positive systems [21]. More recently, probabilistic observers have been formulated for a class of uncertain biological processes [5]; these observers take advantage of the knowledge of probability density functions (PDFs) for the uncertain parameters to calculate the PDFs of the unmeasured sate variables.

An application of interval observers to an anaerobic WWTP is presented next; another application to an activated sludge process can be found in [11]. 


\subsubsection{Application to an Anaerobic Digester}

We consider the same reduced model of an anaerobic digestion plant as in subsection 1.4.3. The objective is to design an interval observer that estimates the COD concentration $S_{t}$ from on-line methane measurements, with $\mu_{t}(\cdot)$ and $\alpha$ being unknown.

Using Eq. (1.8) of the methane flow rate, Eq. (1.7) can be rephrased as:

$$
\frac{d S_{t}}{d t}=-\gamma q_{\mathrm{CH}_{4}}-D\left(S_{t}-S_{t}^{\text {in }}\right)
$$

where the uncertain parameter $\gamma=k_{t} / k_{m}$ is such that $\gamma^{-} \leq \gamma \leq \gamma^{+}$; the inlet COD concentration $S_{t}^{\text {in }}(t)$ fluctuates between known bounds as $S_{t}^{\text {in }}{ }^{-}(t) \leq S_{t}^{\text {in }}(t) \leq S_{t}^{\text {in }}+(t), \forall t \geq t_{0}$; and the initial COD concentration is bounded as $S_{t}^{-}\left(t_{0}\right) \leq S_{t}\left(t_{0}\right) \leq S_{t}^{+}\left(t_{0}\right)$.

Property 2 The auxiliary dynamic system

$$
\left\{\begin{array}{l}
\frac{d S_{t}^{-}}{d t}=-\gamma^{+} q_{C H_{4}}-D\left(S_{t}^{-}-S_{t}^{i{ }^{-}}\right) \\
\frac{d S_{t}^{+}}{d t}=-\gamma^{-} q_{C H_{4}}-D\left(S_{t}^{+}-S_{t}^{i{ }^{+}}\right)
\end{array}\right.
$$

is an interval observer for $S_{t}$, i.e. guarantees that $S_{t}^{-}(t) \leq S_{t}(t) \leq S_{t}^{+}(t)$ at each $t \geq t_{0}$.

Proof. It is easily verified that both lower bound $e^{-}=S_{t}-S_{t}^{-}$and upper bound $e^{+}=$ $S_{t}^{+}-S_{t}$ on the observation error remain positive. (See [3] for details.)

In practice, it was found that the upper bound $S_{t}^{+}(t)$ is weak when large uncertainties are considered for $\gamma$ and $S_{t}^{\text {in }}(t)$. These considerations motivate the following improvements.

Improvements The idea for reducing prediction intervals consists of using structured kinetic models for $\mu(\cdot)$, despite uncertainty. We suppose here that the process does not operate in a region where inhibition phenomena occur, and use a Monod kinetic model for obtaining an estimate of $q_{\mathrm{CH}_{4}}$ as

$$
\hat{q}_{\mathrm{CH}_{4}}\left(S_{t}\right)=k_{m} \mu_{t} \frac{S_{t}}{S_{t}+K_{S_{t}}} \hat{X}_{t}
$$


where $\hat{X}_{t}=\frac{S_{t}^{\text {in }}-S_{t}}{\alpha k_{t}}$. Then, these estimates are used in the following robust observer [3],

$$
\frac{d S_{t}}{d t}=-\gamma q_{\mathrm{CH}_{4}}-D\left(S_{t}-S_{t}^{\mathrm{in}}\right)+\lambda\left(q_{\mathrm{CH}_{4}}-\hat{q}_{\mathrm{CH}_{4}}\left(S_{t}\right)\right)
$$

Finally, an interval observer is derived from Eq. (1.11) as

$$
\left\{\begin{array}{l}
\frac{d S_{t}^{-}}{d t}=\phi_{\epsilon}\left(S_{t}^{-}\right)\left[-\gamma q_{\mathrm{CH}_{4}}-D\left(S_{t}^{-}-S_{i n}\right)+\lambda\left(S_{i n}-S_{t}^{-}\right)\left(q_{\mathrm{CH}_{4}}-q_{\hat{\mathrm{CH}}_{4}}{ }^{+}\left(S_{t}^{-}\right)\right)\right] \\
\frac{d S_{t}^{+}}{d t}=\phi_{\epsilon}\left(S_{t}^{+}\right)\left[-\gamma q_{\mathrm{CH}_{4}}-D\left(S_{t}^{+}-S_{i n}\right)+\lambda\left(S_{i n}-S_{t}^{+}\right)\left(q_{\mathrm{CH}_{4}}-q_{\hat{\mathrm{CH}}_{4}}{ }^{-}\left(S_{t}^{+}\right)\right)\right]
\end{array}\right.
$$

where the function $\phi_{\epsilon}\left(S_{t}^{-}\right)=S_{t}^{-} /\left(S_{t}^{-}+\epsilon\right)$ (with $0<\epsilon \ll \inf S_{t}(t)$ ) is used to enforce $S_{t} \geq 0$; and the bounds $\hat{q}_{\mathrm{CH}_{4}}^{-}$and $\hat{q}_{\mathrm{CH}_{4}}^{+}$on $\hat{q}_{\mathrm{CH}_{4}}$ are calculated by considering the bounds $\mu_{t}^{-}$and $\mu_{t}^{+}$on $\mu_{t}$. This observer is applied to a real process in the next paragraph.

Application to Real Measurements The interval observer given in Eq. (1.11) was implemented on a pilot-scale fixed bed up-flow anaerobic digester used for wine wastewater processing. Both the dilution rate $D$ and methane outflow rate $q_{\mathrm{CH}_{4}}$ were measured on-line at a high frequency. Besides on-line measurements, a COD sensor was also used to validate the observer predictions. Details on the plant configuration and the experiments can be found in $[4,22]$. The predictions of the interval observer are presented in Fig. 1.4., together with the off-line COD measurements.
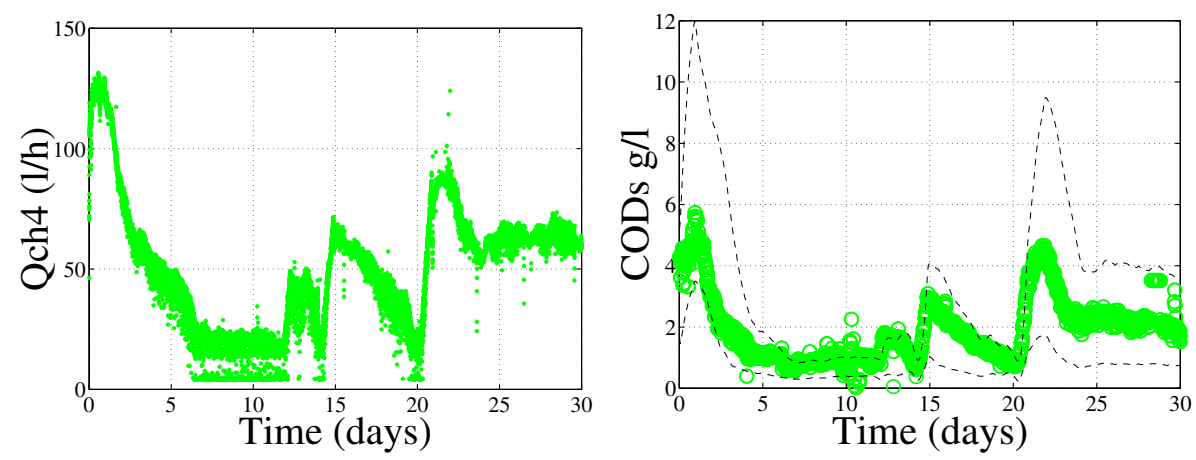

Figure 1.4. Methane flow rate (left plot) and interval observer (- -) for soluble COD (right plot). 


\subsection{CONCLUSIONS}

In this chapter, we have presented a number of methods to design observers for WWTPs. Of course, other techniques exist and we did not pretend to be exhaustive. The observers presented herein assumed constant parameter values in the models. In some cases however, the parameters can evolve during process operation. Algorithms to estimate the parameters must then be used, hence leading to adaptive observers.

For bioprocesses and WWTPs in particular, the type of observer to be considered must be chosen by considering the reliability of the process model at hand, as well as the available measurements. Moreover, designing an observer always results from a trade-off between its robustness towards modeling uncertainties and disturbances, and its convergence rate.

Finally, the implementation of an observer requires a discretization to be performed as regards the continuous-time equations, e.g. an Euler type algorithm. Although not difficult, discretization must be performed carefully. In the case of low measurement frequency, for example, the use of a continuous/discrete observer shall be preferred to full discretization of a continuous-time observer.

To conclude, it is worth insisting on the fact that an observer should always be validated prior to using it in a real treatment plant. In particular, systematic and extensive comparisons should be made between the observer predictions and direct on-site measurements (other than those used to calibrate the observer).

\section{REFERENCES}

1. J. Andrews. A mathematical model for the continuous culture of microorganisms utilizing inhibitory substrate. Biotechnol. Bioengng., 10:707-723, 1968.

2. G. Bastin and D. Dochain. On-line Estimation and Adaptive Control of Bioreactors. Elsevier, Amsterdam, The Netherlands, 1990.

3. O. Bernard and J. L. Gouzé. Closed loop observers bundle for uncertain biotechnological models.

J. Process Contr., 14(7):762-774, 2004. 
4. O. Bernard, M. Z. Hadj-Sadok, D. Dochain, A. Genovesi, and J.-P. Steyer. Dynamical model development and parameter identification for an anaerobic wastewater treatment process. Biotechnol. Bioengng., 75(4):424-438, 2001.

5. B. Chachuat and O. Bernard. Probabilistic observers for a class of uncertain biological processes. in press: Int. J. Robust Nonlinear Control.

6. B. Chachuat, N. Roche, and M. A. Latifi. Reduction of the ASM1 model for optimal control of small-size activated sludge treatment plants. Rev. Sci. Eau, 16(1):5-26, 2003.

7. M. Darouach, M. Zasadzinski, and S. J. Xu. Full-order observers for linear systems with unknown inputs. IEEE Trans. Automat. Contr., 39(3):1068-1072, 1994.

8. J. P. Gauthier and I. Kupka. Deterministic Observation Theory and Applications. Cambridge University Press, New York, 2001.

9. J.-L. Gouzé, A. Rapaport, and M. Z. Hadj-Sadok. Interval observers for uncertain biological systems. Ecol. Modell., 133:45-56, 2000.

10. R. D. Gudi, S. L. Shah, and M. R. Gray. Adaptive multirate state and parameter estimation strategies with application to a bioreactor. AIChE J., 41:2451-2464, 1995.

11. M. Z. Hadj-Sadok and J.-L. Gouzé. Estimation of uncertain models of activated sludge process with interval observers. J. Process Contr., 11(3):299-310, 2001.

12. M. Henze, C. P. L. Grady, W. Gujer, G. v. R. Marais, and T. Matsuo. Activated Sludge Model No. 1. Technical report, IAWQ, London, 1987.

13. M. Hou and P. Müller. Design of observers for linear systems with unknown inputs. IEEE Trans. Automat. Contr., 37(6):871-875, 1991.

14. P. Kudva, N. Viswanadham, and A. Ramakrishna. Observers for linear systems with unknown inputs. IEEE Trans. Automat. Contr., 25(1):113-115, 1980.

15. F. L. Lewis. Optimal Estimation (with an Introduction to Stochastic Control Theory). John Wiley \& Sons, 1986.

16. D. G. Luenberger. Observers for multivariable systems. IEEE Trans. Automat. Contr., 11:190$197,1966$.

17. D. G. Luenberger. Introduction to Dynamic Systems: Theory, Models and Applications. Wiley, 1979. 
18. L.J.S. Lukasse, K. Keesman, and G. van Straten. A recursively identified model for short-term predictions of $\mathrm{NH}_{4} / \mathrm{NO}_{3}$ concentrations in alternating activated sludge processes. J. Process Contr., 9(1):87-100, 1999.

19. A. Rapaport and J.-L. Gouzé. Practical observers for uncertain affine outputs injection systems. In Proc. ECC’99, Karlsruhe, Germany, 1999.

20. A. Rapaport and J.-L. Gouzé. Parallelotopic and practical observers for non-linear uncertain systems. Int. J. Control, 76(3):237-251, 2003.

21. H. L. Smith. Monotone Dynamical Systems: An Introduction to the Theory of Competitive and Cooperative Systems. American Mathematical Society, Providence, Rhode Island, 1995.

22. J.-Ph. Steyer, J.-C. Bouvier, T. Conte, P. Gras, and P. Sousbie. Evaluation of a four year experience with a fully instrumented anaerobic digestion process. Wat. Sci. Technol., 45(45):495-502, 2002.

23. H. Zhao and M. Kümmel. State and parameter estimation for phosphorus removal in an alternating activated sludge process. J. Process Contr., 5(5):341-351, 1995. 